\title{
New opportunity for orphan drug development in Japan: Early exploratory clinical trial bases promote drug translation from basic studies to clinical application
}

\author{
Peipei Song, Jianjun Gao, Norihiro Kokudo, Wei Tang* \\ Department of Surgery, Graduate School of Medicine, The University of Tokyo, Tokyo, Japan.
}

\begin{abstract}
Summary In Japan, although orphan drug legislation has been established in 1993 to encourage drug research and development (R\&D) for intractable and rare diseases, nearly half of the orphan drugs in the Japanese market originated from the European Union (EU) or the United States of America (USA). Availability of orphan drugs for intractable and rare diseases is compounded by the "drug lag" phenomenon, which is mainly caused by the imperfect clinical trial environment in Japan. In recent years, the Japanese government paid great attention to development of innovative drugs and medical devices which originated from Japan. With financial support and institutional guarantees from government, the project of "Early Exploratory Clinical Trial Bases for Specific Research Areas" was launched in 2011 and 5 institutions were selected as the national early exploratory clinical trial bases for specific research areas including cancer, cerebral and cardiovascular diseases, neuropsychiatric disorders, and immunological intractable diseases. The early exploratory clinical trial bases offer a new opportunity for drug development for immunological and neuropsychiatric intractable diseases, thereby promoting orphan drug translation from basic studies to clinical use.
\end{abstract}

Keywords: Intractable and rare diseases, orphan drugs, clinical trial, autoimmune disease, neuropsychiatric disorders

\section{Introduction}

New drug development in Japan is pushed forward by the process of pre-clinical research and clinical trials. After confirmation of safety and effectiveness, pharmaceutical companies can apply to the Ministry of Health, Labour and Welfare (MHLW) for marketing approval. Currently, the time period for drug development in Japan is longer and the number of Japanese originated innovative drugs is fewer compared to that in the European Union (EU) and the United States of America (USA) (1).

Concerning drugs for intractable and rare diseases, orphan drug legislation was established in Japan in 1993 to encourage the corresponding drug research and development (R\&D). The incentives mainly

\section{*Address correspondence to:}

Dr. Wei Tang, Department of Surgery, Graduate School of Medicine, The University of Tokyo, Hongo 7-3-1, Bunkyo-ku, Tokyo, 113-8655, Japan.

E-mail: TANG-SUR@h.u-tokyo.ac.jp include financial subsidies for up to $50 \%$ of expenses for clinical and non-clinical research during the entire research process, exclusive marketing rights for 10 years (compared to 6 years for other medications), 15\% tax credits on research costs excluding financial subsidies, and up to a $14 \%$ reduction in corporate tax $(2,3)$. However, almost half of the orphan drugs in the Japanese market originated from the EU or USA although the orphan drug legislation has been implemented for nearly 20 years (3). Generally speaking, the length of drug research to market cycle is $10-12$ years (4), but there is about a two-year lag period for drug approval in Japan compared with the EU and USA due to the delayed clinical trial application and the relatively longer approval period. This situation is called "drug lag" in Japan. For many drugs originated from Japan, clinical trials are first conducted in the EU or USA, though the basic research has been completed in Japan. Moreover, less drug clinical trials are led by physicians in Japan with a total number of 71 during the period between 2004 to 2010 (5). 
The main cause for drug lag is that the drug clinical trial environment is less than perfect in Japan. First, inadequate cooperation between academic institutions and pharmaceutical companies makes drug R\&D goals not very clear, leading to the lag from basic research to clinical trials. Second, insufficient national support measures and institutional guarantees (involving both physicians and professional clinical institutions) for being first-in-human clinical trials affect the development of drugs in clinical trials, resulting in a lag period of practical applications compared to the EU and USA. If this condition continues, the Japanese patients cannot get the most advanced drugs in a timely fashion and the international competitiveness of Japanese pharmaceutical companies will be weakened, which ultimately influences the improvement of health care levels in Japan.

\section{Early exploratory clinical trial bases for specific research areas}

In recent years, the Japanese government has paid great attention to the development of innovative drugs and medical devices which originated from Japan with the goals of accelerating Japanese patients getting the most advanced medical service as well as enhancing the international competitiveness of Japanese pharmaceutical companies. According to the "New Growth Strategy" and "4th Science and Technology Basic Plan" formulated by the Cabinet Office in 2010 and 2011, the "Life Innovation Project for Constructing Longevity and Healthy Society" was established. Under this project, three government departments including the MHLW, the Ministry of Education, Culture, Sports, Science and Technology (MEXT), and the Ministry of Economy, Trade and Industry (METI) jointly pushed forward development and application of innovative drugs and medical devices for specific research areas. For the specific research areas of cancer, hepatitis, mood disorders, intractable and rare disease, etc., the MHLW invested 13.1 billion yen in 2011 and 18.5 billion yen in 2012 (6,7). Specifically, the project of "Early Exploratory Clinical Trial Bases for Specific Research Areas" was officially launched in 2011 with financial support and institutional guarantees from government.

The early exploratory clinical trials for drugs characterized by early small-scale, studies in humans, and exploratory purposes, are conducted through the phases of "First in Human" and "Proof of Concept" to validate drug safety and efficacy. Data from early exploratory clinical trials will be conducive to the next phase of clinical trials and will accelerate the development of other medications for similar indications. In order to promote the development of innovative drugs and medical devices originated from Japan, the Japanese government launched the project of "Early Exploratory Clinical Trial Bases for Specific Research Areas" and initiated public bidding for domestic medical institutions in 2011. Forty one institutions submitted their research plans according to the bidding requirements. After two rounds of screening, 5 institutions were finally determined as the national early exploratory clinical trial bases for the specific research areas of cancer, cerebral and cardiovascular diseases, neuropsychiatric disorders, and immunological intractable diseases (Table 1). For each base, the Japanese government invests up to 500 million yen for infrastructure construction and 150 million yen for clinical trial research led by physicians (8).

\section{The new opportunity for drug development for intractable and rare diseases}

The confirmed 5 national early exploratory clinical trial bases include 4 bases for drugs and 1 base for medical devices. Of the 4 drug bases, 2 bases have made clear plans for development of new drugs against intractable and rare diseases.

The early exploratory clinical trial base for immunological intractable diseases The School of Medicine, Keio University was confirmed as the national early exploratory clinical trial base for immunological intractable diseases. The Keio University Hospital (KUH) is one of the representative hospitals for treatment of immunological intractable diseases. Established in 2010, Keio University Hospital Immunology Integrated Medical Care Center has conducted several studies concerning diagnosis and treatment of immunological intractable diseases such as Crohn's disease, ulcerative colitis, systemic lupus erythematosus, and rheumatoid arthritis $(9,10)$. Since it was selected as a national early exploratory clinical trial base in 2011, KUH has strengthened construction of infrastructure facilities with support of governmental finance and policy under the mission of R\&D of novel drugs for patients with immunological intractable diseases. Meanwhile, the base

Table 1. National early exploratory clinical trial bases for specific research areas in Japan

\begin{tabular}{lll}
\hline Institution & Type & Research Area \\
\hline National Cancer Center Hospital East & Drug & Cancer \\
Osaka University Hospital & Drug & Cerebral and cardiovascular disease \\
National Cerebral and Cardiovascular Center & Medical device & Cerebral and cardiovascular disease \\
The University of Tokyo Hospital & Drug & Neuropsychiatric disorder \\
School of Medicine, Keio University & Drug & Immunological intractable disease \\
\hline
\end{tabular}


cooperates with other departments of Keio University such as the pharmaceutical supervision lab and clinical drug evaluation lab in the School of Pharmaceutics in order to construct a comprehensive system containing drug safety, efficacy, and supervision evaluation.

The early exploratory clinical trial base for neuropsychiatric intractable diseases The University of Tokyo Hospital (UTH) was confirmed as the national early exploratory clinical trial base for neuropsychiatric disorders. One of the major tasks is to promote development of drugs for Alzheimer's disease (AD), which is one of the neuropsychiatric intractable diseases. With society aging, the number of patients afflicted by $\mathrm{AD}$ has increased. However, there are no drugs for curing this disease thus far. This situation is compounded by lack of quantifiable methods for evaluation of drug efficacy. Since 2008, in working towards a diseasemodifying therapy for AD, The University of Tokyo has carried out studies for the Japanese AD Neuroimaging Initiative (J-ADNI) and explored quantification for evaluation of AD drugs by using brain imaging technology such as positron emission tomographycomputed tomography (PET-CT) $(11,12)$. Considering the rate of disease progression and the invisibility of drug efficacy, it is necessary to conduct the trials in patients in the early phase of AD. Applying the J-ADNI technology, doctors in UTH managed clinical trials to confirm the rate of disease progression and the efficacy of drug treatment. Since being confirmed as a national early exploratory clinical trial base in 2011, the base is dedicated to construct infrastructure facilities including human resources and equipments with support of governmental finance and policy. The first-in-human clinical trials in healthy volunteers is programmed to be conducted in 2013 and clinical trials in patients in the early phase of $\mathrm{AD}$ or mild cognitive impairment will be carried out in 2014 after confirmation of drug safety and clarification of pharmacodynamics.

\section{Conclusion}

A drug lag phenomenon exists in drug R\&D in Japan. The main cause of this phenomenon is that the environment for drug clinical trial bases is not perfect, which leads to delay of clinical studies compared with the EU and USA. In 2011, the project of "Early Exploratory Clinical Trial Bases for Specific Research Areas" was launched with the support of the MHLW, MEXT, and METI. Five health institutions were selected as the national early exploratory clinical trial base for specific research areas, which provides opportunities for orphan drug R\&D. With the support of governmental finance and policy, novel drugs targeting immunological and neuropsychiatric intractable diseases will enter into early exploratory clinical trials at the bases of KUH and UTH. Cooperation among government, basic research institutions and pharmaceutical companies will promote the process of drug translation from basic studies to clinical application, accelerate the availability of drugs for intractable and rare diseases in Japan, and enhance the international competitiveness of Japan original medicines, thereby increasing the medical level of intractable and rare diseases treatment in Japan.

\section{Acknowledgement}

This work was supported by the International Research and Cooperation Association for Bio \& Socio-Sciences Advancement Group for Rare Diseases Research.

\section{References}

1. Tsuji K, Tsutani K. Personal imports of drugs to Japan in 2005 - An analysis of import certificates. J Clin Pharm Ther. 2008; 33:545-552.

2. Song PP, Gao JJ, Inagaki Y, Kokudo N, Tang W. Intractable and rare diseases research in Asia. BioSci Trends. 2012; 6:48-51.

3. Song PP, Gao JJ, Inagaki Y, Kokudo N, Tang W. Rare diseases, orphan drugs, and their regulation in Asia: Current status and future perspectives. Intractable Rare Dis Res. 2012; 1:3-9.

4. Tambuyzer E. Rare diseases, orphan drugs and their regulation: Questions and misconceptions. Nat Rev Drug Discov. 2010; 9:921-929.

5. Pharmaceuticals and Medical Devices Agency. The Bussiness Report in 2010. http://www.pmda.go.jp/guide/ outline/report/report_22.html (accessed April 5, 2012).

6. The Ministry of Health, Labour and Welfare. The Explanatory Material of the Ministry of Health, Labour and Welfare Ministerial Conference. http://www.mhlw. go.jp/topics/2011/01/dl/tp0119-1_13.pdf(accessed April 8, 2012).

7. The Ministry of Health, Labour and Welfare. The List of Budget on Priority Measures in 2012. http://www.mhlw. go.jp/wp/yosan/yosan/12syokan/dl/25.pdf (accessed April 9, 2012).

8. The Ministry of Health, Labour and Welfare. The Results of Early Exploratory Clinical Trial Bases for Specific Research Areas. http://www.mhlw.go.jp/stf/houdou/ 2r9852000001jym4.html (accessed March 25, 2012).

9. Miyoshi J, Yajima T, Okamoto S, Matsuoka K, Inoue N, Hisamatsu T, Shimamura K, Nakazawa A, Kanai T, Ogata H, Iwao Y, Mukai M, Hibi T. Ectopic expression of blood type antigens in inflamed mucosa with higher incidence of FUT2 secretor status in colonic Crohn's disease. J Gastroenterol. 2011; 46:1056-1063.

10. Takeuchi T, Suzuki K, Kondo T, Yoshimoto K, Tsuzaka $\mathrm{K} . \mathrm{CD} 3 \zeta$ defects in systemic lupus erythematosus. Ann Rheum Dis. 2012; 71 (Suppl 2):i78-i81.

11. Iwatsubo T. Towards the disease-modifying therapy of Alzheimer's Disease: Current status of J-ADNI. The Journal of the Proceeding of the Annual Meeting of the Japanese Research Group on Senile Dementia. 2011; 18:125-126. (in Japanese)

12. Iwatsubo T. Japanese Alzheimer's Disease Neuroimaging Initiative: Present status and future. Alzheimers Dement. 2010; 6:297-299.

(Received March 1, 2012; Accepted March 9, 2010) 